\title{
Compact Quantum Groups of Face Type
}

\author{
By
}

\author{
Takahiro HAYASH ${ }^{*}$
}

\begin{abstract}
We give a generalization of the compact quantum group theory of S. Woronowicz, or rather its variant due to T. Koornwinder and M. Dijkhuizen. Our framework covers algebras of $L$-operators of lattice models of face type without spectral parameters and "Galois quantum group" of AFD II $_{1}$-subfactors of index $<4$.
\end{abstract}

\section{Introduction}

In [H4], inspired by the quantum inverse scattering method and solvable lattice models of face type, we introduced a new class of quantum groups, which is called the class of face algebras. It contains all bialgebras as a subclass. Moreover, many important concepts in the bialgebra theory - such as antipodes, universal $R$-matrixes and group-like elements - have counterparts in the theory of face algebras.

In this paper, motivated to combine our theory with Jones' index theory, we study face algebras with some additional properties, which we call compact Hopf face algebras. We give abstract harmonic analytic results of the algebras, including the positivity of Haar functionals and the existence of certain linear functionals $\mathbf{Q}^{Z}$ which describe both the modular properties of the Haar functionals and the squares of the antipodes. The functionals $\mathbf{Q}^{Z}$ may be viewed as a variant of Woronowicz's functional $f_{Z}$ which appears in his theory of compact matrix pseudogroups (cf. [W]). In successive paper [H5], we apply the results of this paper to give a "Galois correspondence" between irreducible comodules of compact Hopf face algebras and a certain class of irreducible AFD $\mathrm{II}_{1}$-subfactors, which includes all of those of index $<4$.

Actually, instead of Woronowicz's original approach, we generalize a new approach [K] due to Koornwinder and Dijkhuizen. Roughly speaking, Woronowicz's theory deals with $*$-Hopf algebras whose representations are

Communicated by T. Miwa, September 12, 1995.

1991 Mathematics Subject Classification(s): 16W30, 81R50

* Department of Mathematics, School of Science, Nagoya University, Chikusa-ku, Nagoya 464, Japan. 
unitary, while the theory of Koornwinder and Dijkhuizen deals with those whose corepresentations are unitary. From another point of view, one may say that the former studies the "algebra of continuous functions" on quantum group $G$, while the latter studies the algebra of "polynomial functions" on $G$. Hence the latter is more algebraic and easier than the former. And more significantly, the latter is more suitable for our examples.

In Section 1 and Section 2, we recall some elementary results on face algebras and split cosemisimple coalgebras respectively. In Section 3, we study face algebras which are split cosemisimple as coalgebras and give some results on their Haar functionals. In Section 4, we study compact Hopf face algebras and give results mentioned above. In Section 5, we show that the dual of a finite-dimensional compact Hopf face algebra is again compact. In Section 6, we give some examples of compact Hopf face algebras and give explicit formulas of $\mathbb{Q}^{Z}$. In Section 7, we give a useful result to show the existence of an antipode of a face algebra.

I would like to thank Prof. M. Noumi for his information about Koornwinder's work [K].

The coproduct and the counit of a coalgebra $C$ will be denoted by $\Delta$ and $\varepsilon$ respectively, and the sigma notation $\Delta(a)=\sum_{(a)} a_{(1)} \otimes a_{(2)}(a \in C)$ will be used frequently (cf. [S]). We also denote the coaction of a right $C$-comodule $M$ by $u \mapsto \sum_{(u)} u_{(0)} \otimes u_{(1)}(u \in M)$.

\section{§1. Face Algebras}

Let $\mathfrak{S}$ be an algebra over a field $\mathbb{K}$ equipped with a coalgebra structure $(\mathfrak{H}, \Delta, \varepsilon)$. Let $\mathscr{V}$ be a finite set and let $\left\{e\left(\begin{array}{l}i \\ j\end{array}\right) \mid i, j \in \mathscr{V}\right\}$ be elements of $\mathfrak{H}$. We say that $\mathfrak{S}=\left(\mathfrak{S},\left\{e\left(\begin{array}{l}i \\ j\end{array}\right)\right\}\right)$ is a $\mathscr{V}$-face algebra if the following conditions are satisfied for each $a, b \in \mathfrak{S}$ and $i, j, i^{\prime}, j^{\prime} \in \mathscr{V}$ :

$$
\begin{aligned}
& \Delta(a b)=\Delta(a) \Delta(b), \\
& e\left(\begin{array}{l}
i \\
j
\end{array}\right) e\left(\begin{array}{l}
i^{\prime} \\
j^{\prime}
\end{array}\right)=\delta_{i i^{\prime}} \delta_{j j^{\prime}} e\left(\begin{array}{l}
i \\
j
\end{array}\right), \sum_{k, \ell \in \mathscr{V}} e\left(\begin{array}{l}
k \\
\ell
\end{array}\right)=1, \\
& \Delta\left(e\left(\begin{array}{c}
i \\
j
\end{array}\right)\right)=\sum_{k \in \mathcal{V}} e\left(\begin{array}{l}
i \\
k
\end{array}\right) \otimes e\left(\begin{array}{l}
k \\
j
\end{array}\right), \varepsilon\left(e\left(\begin{array}{l}
i \\
j
\end{array}\right)\right)=\delta_{i j}, \\
& \sum_{k \in \mathscr{V}} \varepsilon\left(a e_{k}\right) \varepsilon\left(\stackrel{\circ}{e}_{k} b\right)=\varepsilon(a b) .
\end{aligned}
$$

Here, in the final axiom, we use the notation; 


$$
\stackrel{\circ}{e}_{i}=\sum_{j \in \mathscr{V}} e\left(\begin{array}{l}
i \\
j
\end{array}\right), \quad e_{j}=\sum_{i \in \mathscr{V}} e\left(\begin{array}{l}
i \\
j
\end{array}\right) .
$$

We say that a subspace $\mathfrak{I}$ of a $\mathscr{V}$-face algebra $\mathfrak{H}$ is a biideal if it is both an ideal and a coideal of $\mathfrak{H}$. For a biideal $\mathfrak{I}$ of $\mathfrak{H}$, the quotient $\mathfrak{H} / \mathfrak{I}$ naturally becomes a $\mathscr{V}$-face algebra. A $\mathscr{V}$-face algebra becomes a bialgebra if and only if $\#(\mathscr{V})=1$.

Example 1.1. Let $\mathscr{G}=(\mathscr{V}, \mathscr{E})$ be a finite oriented graph. We denote the source (start) and the range (end) of an edge $\mathbf{p}$ of $\mathscr{G}$ by $\lrcorner(\mathbf{p})$ and $\iota(\mathbf{p})$ respectively. For $r>0$, let $\mathscr{G}^{r}=\amalg_{i, j \in \mathfrak{V}} \mathscr{G}_{i j}^{r}$ be the set of paths on $\mathscr{G}$ of length $r$. That is, $\mathbb{p} \in \mathscr{G}_{i j}^{r}$ if $\mathbf{p}$ is a sequence $\left(\mathbf{p}_{1}, \cdots, \mathbf{p}_{\mathbf{r}}\right)$ of edges of $\mathscr{G}$ such that $\varsigma(\mathbf{p}):=\varsigma\left(\mathbf{p}_{1}\right)=i, \imath\left(\mathbf{p}_{1}\right)=\varsigma\left(\mathbf{p}_{2}\right), \cdots, \imath\left(\mathbf{p}_{r-1}\right)=\varsigma\left(\mathbf{p}_{r}\right), \imath(\mathbf{p}):=\imath\left(\mathbf{p}_{r}\right)=j$. We also define $\mathscr{G}^{0}=\amalg_{i, j \in \mathscr{V}} \mathscr{G}_{i j}^{0}$ by $\mathscr{G}_{i i}^{0}=\{i\}(i \in \mathscr{V})$ and $\mathscr{G}_{i j}^{0}=\varnothing(i \neq j)$. Let $\mathfrak{H}(\mathscr{G})$ be the linear span of the symbols $\left\{e\left(\begin{array}{l}\mathbf{p} \\ \mathbf{q}\end{array}\right) \mid \mathbf{p}, \mathbb{q} \in \mathscr{G}^{r}, r \geq 0\right\}$. Then, $\mathfrak{H}(\mathscr{G})$ becomes a $\mathscr{V}$-face algebra by the following operations:

$$
\begin{aligned}
& e\left(\begin{array}{l}
\mathbf{p} \\
\mathbb{q}
\end{array}\right) e\left(\begin{array}{l}
\mathbf{r} \\
\mathbf{s}
\end{array}\right)=\delta_{\imath(\mathbf{p}), \rho(\mathbf{r})} \delta_{z(\mathbf{q}),\lrcorner(\mathbf{s})} e\left(\begin{array}{l}
\mathbf{p} \cdot \mathbf{r} \\
\mathbf{q} \cdot \mathbf{s}
\end{array}\right), \\
& \Delta\left(e\left(\begin{array}{l}
\mathbf{p} \\
\mathbb{q}
\end{array}\right)\right)=\sum_{\mathfrak{t} \in \mathscr{G} r} e\left(\begin{array}{l}
\mathbf{p} \\
\mathbb{q}
\end{array}\right) \otimes e\left(\begin{array}{l}
\mathbb{t} \\
\mathbf{q}
\end{array}\right), \quad \varepsilon\left(e\left(\begin{array}{l}
\mathbf{p} \\
\mathbb{q}
\end{array}\right)\right)=\delta_{\mathbf{p q}} \quad\left(\mathbf{p}, \mathbb{q} \in \mathscr{G}^{r}, \mathbb{r}, \mathbf{s} \in \mathscr{G}^{s}\right),
\end{aligned}
$$

where, for paths $\mathbf{p}=\left(\mathbf{p}_{1}, \cdots, \mathbf{p}_{r}\right)$ and $\mathbf{r}=\left(\mathbf{r}_{1}, \cdots, \mathbf{r}_{s}\right)$, we set $\mathbf{p} \cdot \mathbf{r}:=\left(\mathbf{p}_{1}, \cdots, \mathbf{p}_{r}\right.$, $\left.\mathbb{r}_{1}, \cdots, \mathbb{r}_{s}\right)$ if $\iota(\mathbf{p})$ coincides with $\sigma(\mathbb{r})$.

See $\S 6$ and $[\mathrm{H} 1-\mathrm{H} 6]$ for more non-trivial examples of face algebras.

Let $S$ be a linear endomorphism on a $\mathscr{V}$-face algebra $\mathfrak{S}$. We say that $S$ is an antipode if it satisfies:

$$
\begin{aligned}
& \sum_{(a)} S\left(a_{(1)}\right) a_{(2)}=\sum_{i \in \mathscr{V}} \varepsilon\left(a e_{i}\right) e_{i} \\
& \sum_{(a)} a_{(1)} S\left(a_{(2)}\right)=\sum_{i \in \mathscr{V}} \varepsilon\left(e_{i} a\right) \stackrel{\circ}{e}_{i} \\
& \sum_{(a)} S\left(a_{(1)}\right) a_{(2)} S\left(a_{(3)}\right)=S(a)
\end{aligned}
$$

for each $a \in \mathfrak{H}$. A $\mathscr{V}$-face algebra is called a $\mathscr{V}$-Hopf face algebra if it has an antipode. When $\#(\mathscr{V})=1$, this definition coincides with the usual one. The antipode is unique if it exists, and it is both an antialgebra and an anticoalgebra endomorphism of $\mathfrak{S}$. Moreover it satisfies 


$$
S\left(e\left(\begin{array}{c}
i \\
j
\end{array}\right) a e\left(\begin{array}{l}
m \\
n
\end{array}\right)\right)=e\left(\begin{array}{l}
n \\
m
\end{array}\right) S(a) e\left(\begin{array}{l}
j \\
i
\end{array}\right) \quad(i, j, m, n \in \mathscr{V}, a \in \mathfrak{H}) .
$$

Lemma 1.2. For a $\mathscr{V}$-face algebra $\mathfrak{H}, a, b \in \mathfrak{H}$ and $i, j, m, n \in \mathscr{V}$, we have the following formulas:

$$
\begin{aligned}
& \varepsilon\left(a e_{i}\right)=\varepsilon\left(a \stackrel{\circ}{e}_{i}\right), \varepsilon\left(e_{i} a\right)=\varepsilon\left(\stackrel{\circ}{e}_{i} a\right), \\
& \sum_{(a)} a_{(1)} \varepsilon\left(e_{i} a_{(2)} e_{j}\right)=e_{i} a e_{j}, \\
& \sum_{(a)} \varepsilon\left(e_{i} a_{(1)} e_{j}\right) a_{(2)}=\stackrel{\circ}{e}_{i} a \stackrel{\circ}{e}_{j}, \\
& \sum_{(a)} e_{i} a_{(1)} e_{j} \otimes a_{(2)}=\sum_{(a)} a_{(1)} \otimes \stackrel{\circ}{e}_{i} a_{(2)} \stackrel{\circ}{e}_{j}, \\
& \Delta\left(\stackrel{\circ}{e}_{i} e_{j} a \stackrel{\circ}{e}_{m} e_{n}\right)=\sum_{(a)} \stackrel{\circ}{e}_{i} a_{(1)} \stackrel{\circ}{e}_{m} \otimes e_{j} a_{(2)} e_{n} .
\end{aligned}
$$

See $[\mathrm{H} 4]$ for a proof of these formulas.

\section{§2. Coalgebras}

In this section, we discuss some basic properties of coalgebras and their comodules, which seems to be well-known for specialists.

Let $C$ be a coalgebra over a field $\mathbb{K}$ and $t=\left[t_{p q}\right]_{p, q \in I}$ be a finite size matrix whose entries are elements of $C$. We say that $t$ is a matrix corepresentation if

$$
\Delta\left(t_{p q}\right)=\sum_{r \in I} t_{p r} \otimes t_{r q}, \varepsilon\left(t_{p q}\right)=\delta_{p q} \quad(p, q \in I) .
$$

By definition, the elements $\left\{e\left(\begin{array}{l}i \\ j\end{array}\right) \mid i, j \in \mathscr{V}\right\}$ of a $\mathscr{V}$-face algebra $\mathfrak{S}$ define a matrix corepresentation of $\mathfrak{S}$. For a finite-dimensional right $\mathbb{C}$-comodule $V=\oplus_{q \in I} \mathbb{K} u_{q}$, we define a matrix corepresentation $t$ by the coaction $u_{q} \mapsto \sum_{p} u_{p} \otimes t_{p q}$ and call it the matrix corepresentation associated with $\left(V,\left\{u_{q}\right\}\right)$. Let $s$ be a matrix corepresentation associated with $\left(W,\left\{v_{s}\right\}_{s \in J}\right)$ and let $A: V \rightarrow W ; u_{p} \mapsto \sum_{q} A_{q p} v_{q}$ be a linear map. Then $A$ is a $C$-comodule map if and only if $A t=s A$ in $\operatorname{Mat}(J \times I, C)$.

A coalgebra $C$ is called cosemisimple if there is a coalgebra decomposition $C \simeq \oplus_{\lambda \in \Lambda} C_{\lambda}$ such that each $C_{\lambda}$ has no nontrivial subcoalgebras. If, in addition, each $C_{\lambda}$ is isomorphic to Mat $\left(n_{\lambda}\right)^{*}$ for some $n_{\lambda} \in \mathbb{Z}_{>0}$, then $C$ is called split cosemisimple. It is easy to see that $C$ is split cosemisimple if and 
only if there exists a linear basis $\left\{t_{p q}^{\lambda} \mid \lambda \in \Lambda, p, q \in I(\lambda)\right\}$ such that $t^{\lambda}=\left[t_{p q}^{\lambda}\right]_{p, q \in I(\lambda)}$ is a matrix corepresentation for each $\lambda \in \Lambda$. Moreover the comodules $L(\lambda)=\bigoplus_{q} \mathbb{K} u_{q}^{\lambda}$ defined by $u_{q}^{\lambda} \mapsto \sum_{p} u_{p}^{\lambda} \otimes t_{p q}^{\lambda}$ give complete representatives of irreducible comodules. If the ground field $\mathbb{K}$ is algebraically closed, then $C$ is cosemisimple if and only if it is split cosemisimple (see [L, Lemma 1.2]).

Let $C$ be a coalgebra over the complex number field $\mathbb{C}$ and let $\times: C \rightarrow C ; c \mapsto c^{\times}(c \in C)$ be an antilinear map. We say that $\times$ is a costar structure of $C$ (or, $C$ is a costar coalgebra) if

$$
\left(c^{\times}\right)^{\times}=c, \Delta\left(c^{\times}\right)=\sum_{(c)} c_{(2)}^{\times} \otimes c_{(1)}^{\times}
$$

for each $c \in C$. Let $C$ be a costar coalgebra. It follows from the uniqueness of the counit that

$$
\varepsilon\left(a^{\times}\right)=\overline{\varepsilon(a)} \quad(a \in \mathfrak{H}) .
$$

Let $M$ be a finite-dimensional Hilbert space equipped with a right comodule structure of $C$. We say that $M$ is unitary if

$$
\sum_{(u)}\left(u_{(0)} \mid v\right) u_{(1)}=\sum_{(v)}\left(u \mid v_{(0)}\right) v_{(1)}^{\times}
$$

for each $u, v \in M$. If $\left[t_{p q}\right]$ is a matrix corepresentation of $M$ with respect to an orthonormal basis $\left\{u_{q} \mid q \in I\right\}$, then $M$ is unitary if and only if

$$
t_{p q}^{\times}=t_{q p} \quad(p, q \in I) .
$$

We say that a costar coalgebra $C$ is compact if every finite-dimensional right $C$-comodules are unitary for some inner product. For a costar coalgebra $C$, its dual algebra $C^{*}$ becomes a $*$-algebra with a $*$-structure $*$ defined by

$$
\left\langle X^{*}, a\right\rangle=\overline{\left\langle X, a^{\times}\right\rangle} \quad\left(X \in C^{*}, a \in C\right) .
$$

Lemma 2.1. Let $(C, \times)$ be a costar coalgebra.

(1) Let $M$ be a finite-dimensional $C$-comodule with a Hilbert space structure ( | ). Then $M$ is unitary if and only if the map $\pi_{M}: C^{*} \rightarrow \operatorname{End}(M) ; \pi_{M}(X) u=$ $\sum_{(u)} u_{(0)}\left\langle X, u_{(1)}\right\rangle\left(X \in C^{*}, u \in M\right)$ is a *-representation.

(2) If $\operatorname{dim}(C)<\infty$, then $C$ is compact if and only if $C^{*}$ is a $C^{*}$-algebra.

(3) Every unitary $C$-comodules are completely reducible.

(4) Every compact coalgebras are split cosemisimple.

(5) The coalgebra $C$ is compact if and only if it is spanned by entries of matrix corepresentations associated with unitary comodules.

Proof. Part (1) is straightforward. Part (2) and (3) follows from (1) and a standard argument. By (3), each compact coalgebra is cosemisimple. Since $\mathbb{C}$ is algebraically closed, it is also split cosemisimple. Suppose $C$ is spanned 
by entries of matrix corepresentations of unitary comodules. By (3), [A, Theorem 3.1.4] and [A, Corollary 2.4.8], it is cosemisimple and each of its simple component is isomorphic to End $(L)^{*}$ for some irreducible unitary comodule $L$. This implies (5).

\section{§3. Split Cosemisimple Fæce Algebras}

Let $\mathfrak{S}$ be a $\mathscr{V}$-face algebra. We say that $\mathfrak{H}$ is split cosemisimple if it is split cosemisimple as a coalgebra.

Lemma 3.1. For a right $\mathfrak{S}$-module $M$, we have the following decomposition of a vector space:

$$
\begin{aligned}
& M=\bigoplus_{i, j \in \mathscr{V}} M(i, j), \\
& M(i, j):=\left\{\sum_{(u)} u_{(0)} \varepsilon\left(e_{i} u_{(1)} e_{j}\right) \mid u \in M\right\} .
\end{aligned}
$$

Proof. Set $\varepsilon_{i j}(u)=\sum_{(u)} u_{(0)} \varepsilon\left(e_{i} u_{(1)} e_{j}\right)$ for $u \in M$. By (1.11) and (1.2), we obtain $\varepsilon_{i j} \varepsilon_{m n}=\delta_{i m} \delta_{j n} \varepsilon_{i j}$ and $\sum_{i j} \varepsilon_{i j}=\mathrm{id}$. This proves the lemma.

We call the decomposition (3.1) the face space decomposition of $M$.

Proposition 3.2. For a split cosemisimple $\mathscr{V}$-face algebra 5 , we have a unique decomposition $\mathscr{V}=\amalg_{\lambda \in \Lambda_{0}} \mathscr{V}(\lambda)$ such that $e\left(\begin{array}{l}i \\ j\end{array}\right) \neq 0$ if and only if $i, j \in \mathscr{V}(\lambda)$ for some $\lambda \in \Lambda_{0}$. Moreover $\left\{e\left(\begin{array}{l}i \\ j\end{array}\right) \mid \lambda \in \Lambda_{0}, i, j \in \mathscr{V}(\lambda)\right\}$ is a linear basis of a cosemisimple subcoalgebra of $\mathfrak{5}$.

Proof. Let $R=\oplus_{i \in \mathscr{V}} \mathbb{K} e_{i}$ be a right $\mathfrak{S}$-comodule such that $\left[e\left(\begin{array}{l}i \\ j\end{array}\right)\right]_{i, j \in \mathcal{V}}$ is the matrix corepresentation associated with $\left(R,\left\{e_{i}\right\}\right)$. Let $R=\bigoplus_{\lambda \in \Lambda_{0}} L(\lambda)$ be its irreducible decomposition. Since the face space decomposition of $R$ is given by $R=\bigoplus_{i \in \mathscr{V}} R(i, i) ; R(i, i)=\mathbb{K} e_{i}$, that of $L(\lambda)\left(\lambda \in \Lambda_{0}\right)$ is given by $L(\lambda)=\bigoplus_{i \in \mathscr{V}(\lambda)} L(\lambda)(i, i) ; L(\lambda)(i, i)=\mathbb{K} e_{i}$ for some $\mathscr{V}(\lambda) \subset \mathscr{V}$. Thus we get a decomposition $\mathscr{V}=\amalg_{\lambda \in \Lambda_{0}} \mathscr{V}(\lambda)$ such that $L(\lambda)=\operatorname{span}\left\{e_{i} \mid i \in \mathscr{V}(\lambda)\right\}$ for each $\lambda \in \Lambda_{0}$. In particular $L(\lambda) \simeq \mathbb{L}(\mu)$ if and only if $\lambda=\mu$. Hence $e\left(\begin{array}{l}i \\ j\end{array}\right)=0$ unless $i, j \in \mathscr{V}(\lambda)$ for some $\lambda \in \Lambda_{0}$ and $\left\{e\left(\begin{array}{l}i \\ j\end{array}\right) \mid i, j \in \mathscr{V}(\lambda)\right\}$ is a linear basis of a cosimple component of the coalgebra 5 .

Let $\Lambda, L(\lambda)=\bigoplus_{q \in I(\lambda)} \mathbb{K} u_{q}^{\lambda}$ and $t^{\lambda}=\left[t_{p q}^{\lambda}\right]$ be as in $\S 2$. By the above proposition, we can regard $\Lambda_{0}$ as a subset of $\Lambda$. We also set $I(\lambda):=\mathscr{V}(\lambda)$ 
and $t_{i j}^{\lambda}:=e\left(\begin{array}{l}i \\ j\end{array}\right)(i, j \in \mathscr{V}(\lambda))$ for each $\lambda \in \Lambda_{0}$

Theorem 3.3. Let $\mathfrak{H}$ be a split cosemisimple $\mathscr{V}$-face algebra. Then there exists the unique functional $h \in \mathfrak{H}^{*}$ such that

$$
\begin{aligned}
& \sum_{(a)} a_{(1)} h\left(a_{(2)}\right)=\sum_{k \in \mathcal{V}} h\left(\stackrel{\circ}{e}_{k} a\right) \stackrel{\circ}{e}_{k}, \\
& \sum_{(a)} h\left(a_{(1)}\right) a_{(2)}=\sum_{k \in \mathcal{V}} h\left(a e_{k}\right) e_{k}, \\
& h\left(e_{i}\right)=h\left(\dot{e}_{i}\right)=1,
\end{aligned}
$$

for each $a \in \mathfrak{H}$ and $i \in \mathscr{V}$.

Proof. Suppose both $h_{1}$ and $h_{2}$ satisfy the conditions. By computing $\sum_{(a)} h_{1}\left(a_{(1)}\right) h_{2}\left(a_{(2)}\right)$ in two ways, we see that $h_{1}(a)=h_{2}(a)$ for each $a \in \mathfrak{H}$. Next, we show the existence. We define a linear functional $h \in \mathfrak{H}^{*}$ by

$$
h\left(t_{i j}^{\lambda}\right)= \begin{cases}\#(\mathscr{V}(\lambda))^{-1} & \left(\lambda \in \Lambda_{0}, i, j \in \mathscr{V}(\lambda)\right) \\ 0 & \text { (otherwise). }\end{cases}
$$

If $a=t_{p q}^{\lambda}$ for some $\lambda \in \Lambda \backslash \Lambda_{0}$, then both sides of (3.2) is 0 since $\stackrel{\circ}{e}_{k} t_{p q}^{\lambda}=\sum_{r} \varepsilon\left(e_{k} t_{p r}^{\lambda}\right) t_{r q}^{\lambda}$ by (1.12). If $a=e\left(\begin{array}{l}i \\ j\end{array}\right)$, then both sides of (3.2) is $\#(\mathscr{V}(\lambda))^{-1} \stackrel{\circ}{e}_{i}$. Thus $h$ satisfies the first relation. Proof of other formulas is similar.

We call $h$ in the above theorem the Haar functional of $\mathfrak{S}$.

Lemma 3.4. If $\mathfrak{S}$ has the antipode $S$, then we have the following formulas for each $i, j \in \mathscr{V}$ and $a, b \in \mathfrak{H}$ :

$$
\begin{aligned}
& h\left(e\left(\begin{array}{l}
i \\
j
\end{array}\right) a\right)=h\left(a e\left(\begin{array}{l}
i \\
j
\end{array}\right)\right), \\
& \sum_{(b)} b_{(1)} h\left(a b_{(2)}\right)=\sum_{(a)} S\left(a_{(1)}\right) h\left(a_{(2)} b\right), \\
& \sum_{(b)} h\left(a_{(1)} b\right) a_{(2)}=\sum_{(a)} h\left(a b_{(1)}\right) S\left(b_{(2)}\right), \\
& \sum_{(a)} a_{(1)} h\left(a_{(2)} S(b)\right)=\sum_{(b)} h\left(a S\left(b_{(1)}\right)\right) b_{(2)}, \\
& \sum_{(a)} a_{(1)} h\left(S\left(a_{(2)}\right) b\right)=\sum_{(b)} h\left(S(a) b_{(1)}\right) b_{(2)} .
\end{aligned}
$$


Proof. The first relation easily follows from (1.11), (1.12) and the explicit formula (3.5). Using (1.6), (1.12) and (1.13), we compute

$$
\begin{aligned}
& \sum_{(a)} \sum_{(b)} S\left(a_{(1)}\right) a_{(2)} b_{(1)} h\left(a_{(3)} b_{(2)}\right) \\
= & \sum_{(b)} \sum_{i \in \mathscr{V}} e_{i} b_{(1)} h\left(a \stackrel{\circ}{i}_{i} b_{(2)}\right) \\
= & \sum_{(b)} b_{(1)} h\left(a b_{(2)}\right) .
\end{aligned}
$$

On the other hand, by (3.2), (1.9) and (1.13), the left hand side becomes

$$
\begin{aligned}
& \sum_{(a)} \sum_{i \in \mathscr{V}} S\left(a_{(1)}\right) h\left(\stackrel{\circ}{e}_{i} a_{(2)} b\right) \stackrel{\circ}{e}_{i} \\
= & \sum_{(a)} S\left(a_{(1)}\right) h\left(a_{(2)} b\right) .
\end{aligned}
$$

This proves (3.7). Proof of other formulas are similar. For example, (3.9) is proved by computing the following in two ways:

$$
\sum_{(a)(b)} \sum_{(1)} h\left(a_{(2)} S\left(b_{(1)}\right)\right) S\left(b_{(2)}\right) b_{(3)} .
$$

Proposition 3.5. (cf. [L]) For each $\lambda \in \Lambda$, there exists a matrix $Q^{\lambda} \in \mathrm{GL}(I(\lambda), \mathbb{K})$ satisfying the following relations:

$$
\begin{aligned}
& \operatorname{Tr}\left(Q^{\lambda}\right), \operatorname{Tr}\left(\left(Q^{\lambda}\right)^{-1}\right) \neq 0 \\
& h\left(t_{r s}^{\lambda} S\left(t_{p q}^{\mu}\right)\right)=\frac{1}{\operatorname{Tr}\left(Q^{\lambda}\right)} \delta_{\lambda \mu} \delta_{r q} Q_{p s}^{\lambda} \\
& h\left(S\left(t_{r s}^{\lambda}\right) t_{p q}^{\mu}\right)=\frac{1}{\operatorname{Tr}\left(\left(Q^{\lambda}\right)^{-1}\right)} \delta_{\lambda \mu} \delta_{r q}\left(\left(Q^{\lambda}\right)^{-1}\right)_{p s} \\
& Q^{\lambda} t^{\lambda}=S^{2}\left(t^{\lambda}\right) Q^{\lambda}
\end{aligned}
$$

Here $S^{2}\left(t^{\lambda}\right)$ denotes the matrix corepresentation $\left[S^{2}\left(t_{p q}^{\lambda}\right)\right]_{p q}$. The matrix $Q^{\lambda}$ is unique up to constant factor. If $\lambda \in \Lambda_{0}$, then $Q^{\lambda}$ is a constant matrix.

Proof (cf. [K]). Substituting $a=t_{p q}^{\lambda}$ and $b=t_{r u}^{\mu}$ in (3.9), we obtain

$$
A^{(q r)} t^{\mu}=t^{\lambda} A^{(q r)}
$$

where $A^{(q r)}=A^{(q r)}(\lambda, \mu)$ denotes a matrix defined by

$$
A_{p s}^{(q r)}=h\left(t_{p q}^{\lambda} S\left(t_{r s}^{\mu}\right)\right)
$$


Hence $A^{(q r)}$ defines an $\mathfrak{H}$-comodule map from $L(\mu)$ into $L(\lambda)$. Since $\mathfrak{H}$ is split cosemisimple, each irreducible $\mathfrak{S}$-comodule is absolutely irreducible. Therefore we have

$$
A_{p s}^{(q r)}(\lambda, \mu)=\delta_{\lambda \mu} \delta_{p s} \alpha_{q r}^{\lambda}
$$

for some constant $\alpha_{q r}^{\lambda}$. Using (1.7) and (3.4), we also see that $\sum_{q} \alpha_{q q}^{\lambda}=1$. Next, substituting $a=t_{r u}^{\lambda}$ and $b=S\left(t_{p q}^{\lambda}\right)$ in (3.8), we obtain

$$
\tilde{A}^{(q r)} t^{\lambda}=S^{2}\left(t^{\lambda}\right) \tilde{A}^{(q r)}
$$

where

$$
\tilde{A}_{p s}^{(q r)}:=h\left(t_{r s}^{\lambda} S\left(t_{p q}^{\lambda}\right)\right)=\delta_{q r} \alpha_{s p}^{\lambda} .
$$

Since $\operatorname{Tr}\left(\tilde{A}^{(q r)}\right)=\delta_{q r} \sum_{p} \alpha_{p p}^{\lambda}=\delta_{q r}$, we have $\tilde{A}^{(q q)} \neq 0$. Hence there exists an isomorphism $Q^{\lambda}$ from $L(\lambda)$ onto the comodule which corresponds to $S^{2}\left(t^{\lambda}\right)$. Since $L(\lambda)$ is absolutely irreducible, $Q^{\lambda}$ is unique up to nonzero constant factor. Hence we have $\tilde{A}^{(q r)}=\tilde{\alpha}_{(q r)} Q^{\lambda}$ for some $\tilde{\alpha}_{(q r)} \in \mathbb{K}$. Taking the trace of this equality, we obtain $\tilde{\alpha}_{(q r)}=\delta_{q r} \operatorname{Tr}\left(Q^{\lambda}\right)^{-1}$. Thus we get the relation (3.12) together with (3.14). The last assertion of the proposition follows easily from (3.5), (1.2), (1.9) and (3.12). Proof of other formulas is similar.

Corollary 3.6. Let $\mathfrak{H}$ be a split cosemisimple Hopf face algebra.

(1) The antipode $S$ of $\mathfrak{H}$ is bijective.

(2) The Haar functional $h$ satisfies $h(S(a))=h(a)(a \in \mathfrak{H})$.

Proof. By (3.14), $\left\{S^{2}\left(t_{p q}^{\lambda}\right) \mid p, q \in I(\lambda)\right\}$ is a linear basis of $\operatorname{span}\left\{t_{p q}^{\lambda} \mid p\right.$, $q \in I(\lambda)\}$. This proves (1). Part (2) follows easily from (1) and the uniqueness of the Haar functional.

\section{§4. Compact Hopf Face Algebras}

Definition 4.1. Let $\mathfrak{H}$ be a $\mathscr{V}$-face algebra over $\mathbb{C}$ and $\times: \mathfrak{H} \rightarrow \mathfrak{H}$ a costar structure of the underlying coalgebra of $\mathfrak{H}$. We say that $\times$ is a costar structure of the face algebra $\mathfrak{H}$ (or $\mathfrak{H}=(\mathfrak{H}, \times)$ is a costar face algebra) if the following conditions are satisfied:

$$
\begin{aligned}
& e_{i}^{\times}=\stackrel{\circ}{e}_{i} \quad(i \in \mathscr{V}), \\
& (a b)^{\times}=a^{\times} b^{\times} \quad(a, b \in \mathfrak{H}) .
\end{aligned}
$$

If, in addition, $\mathfrak{H}$ is compact as a coalgebra, then $\mathfrak{H}$ is called a compact face algebra. We say that a biideal $\mathfrak{I}$ of a costar face algebra is a costar biideal if it satisfies $a^{\times} \in \mathfrak{I}$ for each $a \in \mathfrak{I}$.

Lemma 4.2. For a costar face algebra $\mathfrak{H}$, we have the following:

(1) If $\mathfrak{H}$ has an antipode $S$, then it is bijective and $S\left(a^{\times}\right)^{\times}=S^{-1}(a)(a \in \mathfrak{H})$. 
(2) For a costar biideal $\mathfrak{I}, \mathfrak{H} / \mathfrak{I}$ naturally becomes a costar face algebra. If $\mathfrak{H}$ is compact, then so is $\mathfrak{H} / \mathfrak{I}$.

Proof. To show (1), we define an endomorphism $S_{-}$on $\mathfrak{S}$ by $S_{-}(a)=S\left(a^{\times}\right)^{\times}(a \in \mathfrak{H})$. Using (4.2) and (2.3), we compute

$$
\begin{aligned}
\sum_{(a)} a_{(2)} S_{-}\left(a_{(1)}\right) & =\left(\sum_{(a)} a_{(2)}^{\times} S\left(a_{(1)}^{\times}\right)\right)^{\times} \\
& =\left(\sum_{\left(a^{\times}\right)}\left(a^{\times}\right)_{(1)} S\left(\left(a^{\times}\right)_{(2)}\right)\right)^{\times} \\
& =\sum_{i \in \mathscr{V}} \varepsilon\left(e_{i} a\right) e_{i} .
\end{aligned}
$$

Similarly, we obtain

$$
\sum_{(a)} S_{-}\left(a_{(2)}\right) a_{(1)}=\sum_{i \in \mathcal{V}} \varepsilon\left(a e_{i}\right) \stackrel{\circ}{i}_{i}, \sum_{(a)} S_{-}\left(a_{(3)}\right) a_{(2)} S_{-}\left(a_{(1)}\right)=S_{-}(a) .
$$

By [H4, Proposition 2.5], these three formulas implies $S_{-}=S^{-1}$. The first assertion of (2) is straightforward and the second assertion follows from Lemma $2.1(5)$.

For a costar $\mathscr{V}$-Hopf face algebra $\mathfrak{S}$, we define an antilinear map $*: \mathfrak{H} \rightarrow \mathfrak{H}$ by

$$
a^{*}=S\left(a^{\times}\right) \quad(a \in \mathfrak{H}) .
$$

Then, the first assertion of the above lemma is rewritten as $\left(a^{*}\right)^{*}=a$. Hence $\mathfrak{H}$ becomes a $*$-algebra with respect to this operation. We also note that $\left(a^{\times}\right)^{\times}=a$ implies so-called Woronowicz's condition $S\left(S(a)^{*}\right)^{*}=a$.

The proof of the following two results is quite similar to that of Proposition 2.7 and Theorem 2.8 of Koornwinder [K]. So we omit it.

Proposition 4.3. Let $\mathfrak{S}$ be a compact Hopf face algebra and let $t^{\lambda}$ be the matrix corepresentation associated with an irreducible unitary comodule $L(\lambda)$ and its orthonormal basis $\left\{u_{q}^{\lambda} \mid q \in I(\lambda)\right\}$. Then there exists the unique positive matrix $Q^{\lambda} \in \mathrm{GL}(I(\lambda), \mathbb{C})$ which satisfies the conditions of Proposition 3.5 together with

$$
\operatorname{Tr}\left(Q^{\lambda}\right)=\operatorname{Tr}\left(\left(Q^{\lambda}\right)^{-1}\right) .
$$

The conditions (3.14) and (4.4) completely determine the positive matrix $Q^{\lambda}$. For $\lambda \in \Lambda_{0}, Q^{\lambda}$ is an identity matrix.

Theorem 4.4. The Haar functional $h$ of a compact Hopf face algebra $\mathfrak{H}$ is positive faithful, that is, $h\left(a^{*} a\right)>0$ for each $a \in \mathfrak{S} \backslash\{0\}$. 
Definition 4.5. For a compact Hopf face algebra $\mathfrak{H}$, we define a linear functional $\mathbf{Q}^{z}(z \in \mathbb{C})$ on $\mathfrak{H}$ by

$$
\mathbf{Q}^{z}\left(t_{p q}^{\lambda}\right)=\left(\left(\mathbf{Q}^{\lambda}\right)^{z}\right)_{p q} \quad(\lambda \in \Lambda, p, q \in I(\lambda))
$$

and call it the Woronowicz functional of $\mathfrak{H}$, where $t^{\lambda}$ and $\mathbf{Q}^{\lambda}$ are as in Proposition 4.3. We also set $\mathbf{Q}=\mathbf{Q}^{1}$.

Theorem 4.6. The Woronowicz functional $\mathbf{Q}^{z}$ of a compact $\mathscr{V}$-Hopf face algebra $\mathfrak{H}$ satisfies the following properties:

(1) For each $a \in \mathfrak{H}$, the function $z \mapsto \mathbf{Q}^{z}(a)$ is an entire analytic function and there are constants $M>0$ and $\mu \in \mathbb{R}$ such that $\left|\mathbf{Q}^{z}(a)\right| \leq M \exp (\mu \Re \mathfrak{R e}(z))$ for each $z \in \mathbb{C}$.

(2) For each finite-dimensional right unitary $\mathfrak{S}$-comodule $M, \pi_{M}(\mathbf{Q})$ is a positive invertible matrix such that $\operatorname{Tr} \pi_{M}(\mathbf{Q})=\operatorname{Tr}\left(\pi_{M}(\mathbf{Q})^{-1}\right)$, where $\pi_{M}: \mathfrak{S}^{*} \rightarrow \operatorname{End}(M)$ is as in Lemma 2.1.

(3) For each $z, w \in \mathbb{C}, a, b \in \mathfrak{H}$ and $i, j \in \mathscr{V}$, we have the following formulas:

$$
\begin{aligned}
& \mathbf{Q}^{0}=\varepsilon, \mathbf{Q}^{z} \mathbf{Q}^{w}=\mathbf{Q}^{z+w}, \\
& \sum_{(a)} \mathbf{Q}\left(a_{(1)}\right) a_{(2)} \mathbf{Q}^{-1}\left(a_{(3)}\right)=S^{2}(a), \\
& h(a b)=\sum_{(a)} \mathbf{Q}\left(a_{(1)}\right) h\left(b a_{(2)}\right) \mathbf{Q}\left(a_{(3)}\right), \\
& \sum_{(a b)} \mathbf{Q}\left((a b)_{(1)}\right)(a b)_{(2)} \mathbf{Q}\left((a b)_{(3)}\right) \\
& =\sum_{(a)} \sum_{(b)} \mathbf{Q}\left(a_{(1)}\right) a_{(2)} \mathbf{Q}\left(a_{(3)}\right) \mathbf{Q}\left(b_{(1)}\right) b_{(2)} \mathbf{Q}\left(b_{(3)}\right), \\
& \mathbf{Q}^{z}(a b)=\sum_{k \in \mathcal{V}} \mathbf{Q}^{z}\left(a e_{k}\right) \mathbf{Q}^{z}\left(\stackrel{\circ}{e}_{k} b\right), \\
& \mathbf{Q}^{z}\left(e_{i} a e_{j}\right)=\mathbf{Q}^{z}\left(\stackrel{\circ}{e}_{i} a e_{j}\right) \\
& \mathbf{Q}^{z}\left(e\left(\begin{array}{l}
i \\
j
\end{array}\right)\right)=\delta_{i j}, \\
& \mathbf{Q}^{z}(S(a))=\mathbf{Q}^{-z}(a), \\
& \left(\mathbb{Q}^{z}\right)^{*}=\mathbf{Q}^{\bar{z}} .
\end{aligned}
$$

Proof. As immediate consequences of the definition of $\mathbf{Q}^{z}$ and Propositions 3.5, 4.3, we obtain (1), (2) and (4.6), (4.7), (4.12), (4.14). Proof of (4.8) and (4.9) is quite similar to that of Woronowicz's original paper [W]. By (4.7), we have 


$$
\sum_{(a)} \mathbf{Q}^{n}\left(a_{(1)}\right) e_{i} a_{(2)} e_{j}=\sum_{(a)} e_{i} S^{2 n}\left(a_{(1)}\right) e_{j} \mathbf{Q}^{n}\left(a_{(2)}\right)
$$

for each $n \in \mathbb{Z}_{>0}$. Substituting both hand sides into the counit and using (1.9) (1.11) and (1.12), we get (4.11) for $z=n \in \mathbb{Z}$. By virtue of Carlson's theorem ([W, Lemma 5.5]), (4.11) holds for any $z \in \mathbb{C}$. To show (4.10), set

$$
\sigma_{n}(a)=\sum_{(a)} \mathbf{Q}^{n}\left(a_{(1)}\right) a_{(2)} \mathbf{Q}^{n}\left(a_{(3)}\right) \quad\left(a \in \mathfrak{H}, n \in \mathbb{Z}_{>0}\right) .
$$

Using (1.12), (4.11), (1.14) and (4.6), we compute

$$
\begin{aligned}
\varepsilon\left(\sigma_{n}(a) e_{i}\right) & =\sum_{(a)} \mathbf{Q}^{n}\left(a_{(1)}\right) \mathbf{Q}^{n}\left(a_{(2)} \stackrel{\circ}{e}_{i}\right) \\
& =\sum_{(a)} \mathbf{Q}^{n}\left(a_{(1)}\right) \mathbf{Q}^{n}\left(a_{(2)} e_{i}\right) \\
& =\mathbf{Q}^{2 n}\left(a e_{i}\right), \\
\varepsilon\left(\stackrel{\circ}{e}_{i} \sigma_{n}(b)\right) & =\mathbf{Q}^{2 n}\left(\circ_{i}^{i} b\right) \quad(a, b \in \mathfrak{H}, i \in \mathscr{V}) .
\end{aligned}
$$

Using these together with (1.4), we get,

$$
\begin{aligned}
& \varepsilon\left(\sigma_{n}(a) \sigma_{n}(b)\right)=\sum_{i \in \mathcal{V}} \mathbf{Q}^{2 n}\left(a e_{i}\right) \mathbf{Q}^{2 n}\left(\stackrel{\circ}{i}_{i} b\right), \\
& \varepsilon\left(\sigma_{n}(a b)\right)=\mathbf{Q}^{2 n}(a b) .
\end{aligned}
$$

Since $\sigma_{n}(a b)=\sigma_{n}(a) \sigma_{n}(b)$ by (4.6), (4.10) holds for $z=2 n \in 2 \mathbb{Z}_{>0}$. Again by Carlson's theorem, (4.10) holds for any $z \in \mathbb{C}$. The proof of (4.13) will be given in $\S 7$.

Proposition 4.7. The relation (4.7) together with the property stated in Theorem 4.6 (2) completely characterizes the Woronowicz functional $\mathbf{Q}=\mathbb{Q}^{1}$.

Proof. This assertion is an immediate consequence of the uniqueness of $\mathbf{Q}^{\lambda}$ stated in Proposition 4.3.

Remark. The Woronowicz functional $\mathbf{Q}^{\mathbf{z}}$ is an analogue of the functional $f_{z}$ of Woronowicz [W]. It will be used to define "QQQ-dimensions" and "Q-traces" of $\mathfrak{H}$-comodules and their endomorphisms (cf. [H5]). This is the reason why we use the letter $\mathbf{Q}$ instead of $f$.

\section{§5. Finite-dimensional Compact Hopf Face Algebras}

Let $\mathfrak{S}$ be a finite-dimensional $\mathscr{V}$-Hopf face algebra and let $\mathfrak{S}^{\circ}$ denote its dual space $\mathfrak{H}^{*}$. We define elements $e\left(\begin{array}{l}i \\ j\end{array}\right)=e_{\mathfrak{H}^{\circ}}\left(\begin{array}{l}i \\ j\end{array}\right)(i, j \in \mathscr{V})$ of $\mathfrak{H}^{\circ}$ by 


$$
\left\langle e\left(\begin{array}{l}
i \\
j
\end{array}\right), a\right\rangle=\varepsilon\left(e_{i} a e_{j}\right) \quad(a \in \mathfrak{H}) .
$$

By $[\mathrm{H} 3, \S 2], \mathfrak{H}^{\circ}$ has a unique structure of $\mathscr{V}$-Hopf face algebra which satisfies :

$$
\begin{aligned}
& \langle X Y, a\rangle=\sum_{(a)}\left\langle X, a_{(1)}\right\rangle\left\langle Y, a_{(2)}\right\rangle, \\
& \langle X, a b\rangle=\sum_{(X)}\left\langle X_{(1)}, a\right\rangle\left\langle X_{(2)}, b\right\rangle, \\
& \langle S(X), a\rangle=\langle X, S(a)\rangle, \\
& \left\langle e\left(\begin{array}{l}
i \\
j
\end{array}\right) X e\left(\begin{array}{c}
m \\
n
\end{array}\right), a\right\rangle=\left\langle X, e\left(\begin{array}{c}
i \\
m
\end{array}\right) a e\left(\begin{array}{l}
j \\
n
\end{array}\right)\right\rangle
\end{aligned}
$$

for each $a, b \in \mathfrak{H}, X, Y \in \mathfrak{H}^{\circ}$ and $i, j, m, n \in \mathscr{V}$. If, in addition, $\mathfrak{H}$ has a costar structure $\times, \mathfrak{H}^{\circ}$ also has a costar structure $\times$ defined by (4.3) and

$$
\left\langle X^{\times}, a\right\rangle=\overline{\left\langle X, a^{*}\right\rangle} \quad\left(X \in \mathfrak{H}^{\circ}, a \in \mathfrak{H}\right) .
$$

It is easy to see that $\left(\mathfrak{S}^{\circ}\right)^{\circ}$ is naturally identified with $\mathfrak{H}$ as a costar face algebra.

Theorem 5.1. (cf. [KP]) If $\mathfrak{H}$ is a finite-dimensional compact $\mathscr{V}$-Hopf face algebra. Then $\mathfrak{H}^{\circ}$ is also compact.

Proof. By Theorem 4.4, $\mathfrak{H}$ becomes a Hilbert space with the inner product $(a \mid b):=h\left(b^{*} a\right) \quad(a, b \in \mathfrak{H})$. It is easy to see that the left regular representation of $\mathfrak{H}$ is a faithful *-representation on $(\mathfrak{H},(\mid))$. Therefore $\mathfrak{S} \simeq\left(\mathfrak{S}^{\circ}\right)^{\circ}$ is a $C^{*}$-algebra. Applying Lemma 2.1 (2) to $C=\mathfrak{H}^{\circ}$, we see that $\mathfrak{H}^{\circ}$ is compact.

Corollary 5.2. Let $\mathfrak{H}$ be a finite-dimensional costar Hopf face algebra. Then $\mathfrak{H}$ is compact if and only if $\mathfrak{H}$ is a $C^{*}$-algebra with respect to the involution * given by (4.3).

Proof. This is an immediate consequence of Lemma 2.1 (2) and the theorem.

Proposition 5.3. Let $\mathfrak{H}$ be a finite-dimensional compact $\mathscr{V}$-Hopf face algebra. If $\mathfrak{H}$ is a bialgebra (i.e. $\#(\mathscr{V})=1$ ), then $\mathbf{Q}^{z}=\varepsilon$ for each $z \in \mathbb{C}$.

Proof. Since $\mathfrak{H}$ is semisimple and cosemisimple, we have $S^{2}=$ id by a result of Larson and Radford [LR]. By the characterization of $Q^{\lambda}$ stated in Proposition 4.3, we get $\mathbf{Q}^{\lambda}=\mathrm{id}$ for each $\lambda \in \Lambda$. This proves the proposition.

\section{§6. Examples}

Let $\mathscr{G}=(\mathscr{V}, \mathscr{E})$ be a connected non-oriented finite graph which has at 
least one edge. We naturally identify $\mathscr{G}$ with an oriented graph $\mathscr{G}^{\prime}$ such that vertex $\left(\mathscr{G}^{\prime}\right)=\mathscr{V}, \#\left(\left(\mathscr{G}^{\prime}\right)_{i j}^{1}\right)=\#$ \{edges of $\mathscr{G}$ between $i$ and $\left.j\right\}$ and that there exists a fixed bijection ${ }^{\sim}:\left(\mathscr{G}^{\prime}\right)_{i j}^{1} \rightarrow\left(\mathscr{G}^{\prime}\right)_{j i}^{1}$ which satisfies $\mathbf{p}^{\sim}=\mathbf{p}\left(\mathbf{p} \in\left(\mathscr{G}^{\prime}\right)_{i j}^{1}\right)$. Let $\mathfrak{H}(\mathscr{G})$ be as in Example 1.1. We define a $\mathscr{V}$-face algebra $\mathfrak{F}(\mathscr{G})$ as a quotient of $\mathfrak{H}(\mathscr{G})$ by the following additional relations:

$$
\begin{aligned}
& \sum_{\mathbf{s} \in \mathscr{G}_{J,-}^{1}} \mu(\imath(\mathbf{s}))^{1 / 2} e\left(\begin{array}{c}
\mathbf{p} \cdot \mathbf{q} \\
\mathbf{s} \cdot \mathbf{s}^{\sim}
\end{array}\right)=\delta_{\mathbf{p}, \mathbf{q} \sim}\left(\frac{\mu(j) \mu(\imath(\mathbf{p}))}{\mu(\supset(\mathbf{p}))}\right)^{1 / 2} e\left(\begin{array}{c}
\supset(\mathbf{p}) \\
j
\end{array}\right), \\
& \sum_{\mathbf{s} \in \mathscr{G}_{J,-}^{1}} \mu(\iota(\mathbf{s}))^{1 / 2} e\left(\begin{array}{c}
\mathbf{s} \cdot \mathbf{s}^{\sim} \\
\mathbf{p} \cdot \mathbf{q}
\end{array}\right)=\delta_{\mathbf{p}, \mathbf{q} \sim}\left(\frac{\mu(j) \mu(\iota(\mathbf{p}))}{\mu(\delta(\mathbf{p}))}\right)^{1 / 2} e\left(\begin{array}{c}
j \\
\supset(\mathbb{p})
\end{array}\right)
\end{aligned}
$$

$\left(\mathrm{p}, \mathbb{q} \in \mathscr{G}^{1}, j \in \mathscr{V}\right)$,

where $\mathscr{G}_{j,-}^{1}:=\amalg_{k \in \mathscr{V}} \mathscr{G}_{j k}^{1}$ and $[\mu(i)]_{i \in \mathscr{V}}$ denotes the Perron-Frobenius eigenvector of the adjacency matrix of $\mathscr{G}$. This example was introduced by the author in [H2] in order to prove a crucial lemma for the classification of $\mathbb{I I}_{1}$-subfactors of Jones' index $<4$. It is also useful for knot theory since it has a structure of "coribbon face algebra."

The face algebra $\mathfrak{F}(\mathscr{G})$ has an antipode $S$ which is given by the following formula (see $\S 7$ ):

$$
S\left(\left(\begin{array}{l}
\mathbb{p} \\
\mathbb{q}
\end{array}\right)\right)=\left(\frac{\mu(\jmath(\mathbb{q})) \mu(\iota(\mathbf{p}))}{\mu(\jmath(\mathbb{p})) \mu(\iota(\mathfrak{q}))}\right)^{1 / 2} e\left(\begin{array}{l}
\mathbb{q}^{\sim} \\
\mathbb{p}^{\sim}
\end{array}\right) \quad\left(\mathbb{p}, \mathbb{q} \in \mathscr{G}^{r}, r \geq 0\right) .
$$

Proposition 6.1. The face algebra $\mathfrak{F}(\mathscr{G})$ is compact with respect to the costar structure given by

$$
e\left(\begin{array}{l}
\mathbf{p} \\
\mathbf{q}
\end{array}\right)^{\times}=e\left(\begin{array}{l}
\mathbb{q} \\
\mathbf{p}
\end{array}\right)
$$

Proof. It is easy to verify that (6.2) gives a well-defined costar structure on $\mathfrak{F}(\mathscr{G})$. Hence the assertion follows from Lemma 2.1 (5).

Proposition 6.2. If $\mathscr{G}$ is one of the Dynkin diagram $A_{n}, D_{n}(n \geq 4)$ or $E_{n}$ $(n=6,7,8)$, then the Woronowicz functional of $\mathfrak{F}(\mathscr{G})$ is given by the following formula:

$$
\mathbb{Q}^{z}(a)=\sum_{i, j \in \mathscr{V}}\left(\frac{\mu(j)}{\mu(i)}\right)^{z} \varepsilon\left(e_{i} a e_{j}\right) \quad(a \in \mathscr{F}(\mathscr{G}), z \in \mathbb{C}) .
$$

Proof. We define a matrix $Q \in \operatorname{Mat}\left(\mathscr{G}^{1}, \mathbb{C}\right)$ by $\mathbb{Q}_{\mathrm{pq}}=\delta_{\mathrm{pq}} \mu(\iota(\mathbb{p})) / \mu(\diamond(\mathrm{p}))$. By direct computation, we get $Q t=S^{2}(t) Q$ and $\operatorname{Tr}(Q)=\operatorname{Tr}\left(Q^{-1}\right)$, where $t=\left[e\left(\begin{array}{l}\mathbb{P} \\ \mathbb{q}\end{array}\right)\right]_{\mathbf{p}, \mathbf{q} \in \mathscr{G}^{1}}$. Hence by the lemma below and the second assertion of Proposition 4.3, we get 


$$
\mathbf{Q}^{z}\left(e\left(\begin{array}{l}
\mathbf{p} \\
\mathbf{q}
\end{array}\right)\right)=\delta_{\mathbf{p q}}\left(\frac{\mu(\iota(\mathbf{p}))}{\mu(\supset(\mathbf{p}))}\right)^{z} \quad\left(\mathbf{p}, \mathbf{q} \in \mathscr{G}^{r}\right)
$$

for $r=1$. Using this, (4.10) and (4.12), we see that (6.4) holds for any $r \geq 0$. This proves the proposition.

Lemma 6.3. If $\mathscr{G}$ is either $A_{n}, D_{n}$ or $E_{n}$, then the $\mathfrak{F}(\mathscr{G})$-comodule $V:=\operatorname{span}\left\{u_{\mathbf{p}} \mid \mathbf{p} \in \mathscr{G}^{1}\right\} ; u_{\mathbf{q}} \mapsto \sum_{\mathbf{p}} u_{\mathbf{p}} \otimes e\left(\begin{array}{l}\mathbf{p} \\ \mathbf{q}\end{array}\right)$ is irreducible.

Proof. By discussions given in $\S 3$ and $\S 4$ of [H2], there exists the unique bilinear pairing $\langle\cdot, \cdot\rangle: \mathfrak{F}(\mathscr{G}) \otimes \mathfrak{F}(\mathscr{G}) \rightarrow \mathbb{C}$ satisfying the following relations:

$$
\begin{aligned}
& \langle a b, c\rangle=\sum_{(c)}\left\langle a, c_{(1)}\right\rangle\left\langle b, c_{(2)}\right\rangle,\langle a, c d\rangle=\sum_{(a)}\left\langle a_{(1)}, c\right\rangle\left\langle a_{(2)}, d\right\rangle, \\
& \left\langle e\left(\begin{array}{l}
i \\
j
\end{array}\right), a\right\rangle=\sum_{i, j \in \mathcal{V}} \varepsilon\left(e_{i} a e_{j}\right)=\left\langle a, e\left(\begin{array}{l}
i \\
j
\end{array}\right)\right\rangle, \\
& \left\langle e\left(\begin{array}{l}
\mathbf{p} \\
\mathbf{q}
\end{array}\right), e\left(\begin{array}{l}
\mathbf{r} \\
\mathbf{s}
\end{array}\right)\right\rangle=W\left(\begin{array}{l}
\mathbf{r} \\
\mathbf{p}
\end{array}\right) \\
& :=\delta_{\mathbf{p r}} \delta_{\mathbf{q s}} \varepsilon+\delta_{\mathbf{p}, \mathbf{s} \sim} \delta_{\mathbf{r}, \mathbf{q} \sim}\left(\frac{\mu(j) \mu(m)}{\mu(i) \mu(n)}\right)^{1 / 2} \varepsilon^{-1} \\
& \left(\mathbf{p} \in \mathscr{G}_{i j}^{1}, \mathbf{q} \in \mathscr{G}_{m n}^{1}, \mathbf{r} \in \mathscr{G}_{i m}^{1}, \mathbf{s} \in \mathscr{G}_{j n}^{1}\right),
\end{aligned}
$$

where $\varepsilon$ denotes a solution of the equation $\varepsilon^{2}+\varepsilon^{-2}+\beta=0$ and $\beta$ denotes the Perron-Frobenius eigenvalue of the adjacency matrix of $\mathscr{G}$. Hence there exists an algebra map $\alpha: \mathfrak{F}(\mathscr{G}) \rightarrow \mathfrak{F}(\mathscr{G})^{*}$ such that $\alpha(a)(b)=\langle a, b\rangle(a, b \in \mathfrak{F}(\mathscr{G}))$. Using

$$
\begin{array}{r}
\pi_{V}\left(\alpha\left(e\left(\begin{array}{l}
\mathbf{p}^{\sim} \\
\mathbf{q}^{\sim}
\end{array}\right)\right)\right) u(\mathbf{p})=W\left(\begin{array}{l}
\mathbf{q}^{\sim} \\
\mathbf{q}^{\sim}
\end{array}\right) u(\mathbf{p}), W\left(\begin{array}{l}
\mathbf{q}_{\mathbf{q}^{\sim}} \\
\mathbf{p}^{\sim}
\end{array}\right) \neq 0 \\
\left(\mathbf{p} \in \mathscr{G}_{i j}^{1}, \mathbf{q} \in \mathscr{G}_{j k}^{1}, i, j, k \in \mathscr{V}\right),
\end{array}
$$

we see that $V$ is irrecucible as a left $\mathfrak{F}(\mathscr{G})^{*}$-module, where $\pi_{V}$ is as in Lemma 2.1. This proves the lemma.

\section{§7. Appendix. Group-like Elements and Antipodes}

In this section, we discuss elementary properties of group-like elements of face algebras. Also, we give a variant of a result of Takeuchi [T] and show the existence of an antipode of $\mathfrak{F}(\mathscr{G})$. We will also apply the result to other examples (cf. [H1, H5, H6]).

Let $g$ be an element of a $\mathscr{V}$-face algebra $\mathfrak{H}$ and $G \in \mathfrak{H}^{*}$ a linear functional 
on $\mathfrak{H}$. We say that $g$ (respectively $G$ ) is group-like if the following relations (7.1) (respectively (7.2)) are satisfied:

$$
\begin{aligned}
& \Delta(g)=\sum_{i \in \mathscr{V}} g e_{i} \otimes g \stackrel{\circ}{e}_{i}, \\
& e\left(\begin{array}{c}
i \\
j
\end{array}\right) g=g e\left(\begin{array}{c}
i \\
j
\end{array}\right)(i, j \in \mathscr{V}), \varepsilon\left(g e_{i}\right)=1 \quad(i \in \mathscr{V}), \\
& G(a b)=\sum_{i \in \mathscr{V}} G\left(a e_{i}\right) G\left(\stackrel{\circ}{e}_{i} b\right) \quad(a, b \in \mathfrak{H}), \\
& G\left(e_{i} a e_{j}\right)=G\left(\stackrel{\circ}{e}_{i} a \stackrel{\circ}{e}_{j}\right)(i, j \in \mathscr{V}), G\left(e_{i}\right)=1 \quad(i \in \mathscr{V}) .
\end{aligned}
$$

We denote by GLE $(\mathfrak{H})$ the set of all group-like elements of $\mathfrak{S}$, and by $\operatorname{GLF}(\mathfrak{H})$ the set of all group-like functionals of $\mathfrak{H}$. By (4.10)-(4.12), the Woronowicz functional $Q^{z}$ of a compact Hopf face algebra is group-like for each $z \in \mathbb{C}$. If $\mathfrak{H}$ is a bialgebra (i.e. $\#(\mathscr{V})=1), G \in \mathfrak{H}^{*}$ is group-like if and only if $G \in \operatorname{Alg}(\mathfrak{S}, \mathbb{K})$.

Proposition 7.1. For a face algebra $\mathfrak{H}, \operatorname{GLE}(\mathfrak{H})$ and $\operatorname{GLF}(\mathfrak{S})$ are multiplicative subsemigroups of $\mathfrak{S} \backslash\{0\}$ and $\mathfrak{H}^{*} \backslash\{0\}$ respectively. If $\mathfrak{H}$ has an antipode, then GLE $(\mathfrak{H})$ and $\mathrm{GLF}(\mathfrak{H})$ are groups and we have $S(g)=g^{-1}$ and $S^{*}(G)=G^{-1}$ for each $g \in \operatorname{GLE}(\mathfrak{S})$ and $G \in \operatorname{GLF}(\mathfrak{S})$.

Proof. The proof of the first assertion is straightforward. Using first two formulas of (7.2), we compute

$$
\begin{aligned}
G\left(\sum_{(a)} S\left(a_{(1)}\right) a_{(2)}\right) & =\sum_{(a)} \sum_{i \in \mathscr{V}} G\left(S\left(a_{(1)}\right) \stackrel{\circ}{e}_{i}\right) G\left(\stackrel{\circ}{e}_{i} a_{(2)}\right) \\
& =\left(S^{*}(G) G\right)(a),
\end{aligned}
$$

where the second equality follows from (1.9) and (1.13). On the other hand, by (1.6) and the third formula of (7.2), the left-hand side of the above formula is equal to $\varepsilon(a)$. This proves $S^{*}(G) G=1$. Similarly, computing $G\left(\sum_{(a)} a_{(1)} S\left(a_{(2)}\right)\right)$ in two ways, we get $G S^{*}(G)=1$. The proof of $S(g)=g^{-1}$ is similar.

Lemma 7.2. For each group-like element $g$ of a $\mathscr{V}$-face algebra $\mathfrak{5}$, $(g-1):=\mathfrak{H}(g-1) \mathfrak{H}$ is a biideal. If $\mathfrak{H}$ has a costar structure $\times$ such that $g^{\times}=g$, then $(g-1)$ is a costar biideal. In particular, $\mathfrak{H} /(g-1)$ is compact if $\mathfrak{S}$ is compact and $g^{\times}=g$.

Proof. The first assertion follows from

$$
\Delta(g-1)=\sum_{i \in \mathscr{V}}\left((g-1) e_{i} \otimes g \dot{e}_{i}+e_{i} \otimes(g-1) \stackrel{\circ}{i}_{i}\right)
$$




$$
\varepsilon(a(g-1) b)=\sum_{i, j \in \mathscr{V}} \varepsilon\left(a e_{i}\right) \varepsilon\left(e_{i}(g-1) e_{j}\right) \varepsilon\left(e_{j} b\right)=0 \quad(a, b \in \mathfrak{H}) .
$$

The second assertion is obvious and the third assertion follows from Lemma $4.2(2)$.

Let $\mathfrak{H}(\mathscr{G})$ be a $\mathscr{V}$-face algebra defined as in Example 1.1. Let $\mathscr{R}=\amalg_{r \geq 0} \mathscr{R}_{r}, \mathscr{R}_{r} \subset$ Mat $\left(\mathscr{G}^{r}, \mathbb{K}\right)$ be a set of matrixes such that $R_{\mathbf{p q}}=0$ for each $R \in \mathscr{R}_{r}$ and $\mathbf{p}, \mathbf{q} \in \mathscr{G}^{r}$ unless $\lrcorner(\mathbf{p})=\varsigma(\mathbf{q})$ and $\iota(\mathbf{p})=\imath(\mathbf{q})$. Since

$$
\mathfrak{I}=\left\langle\sum_{\mathbf{t} \in \mathscr{G}^{r}}\left(R_{\mathbf{p t}} e\left(\begin{array}{l}
\mathbf{t} \\
\mathbf{q}
\end{array}\right)-e\left(\begin{array}{l}
\mathbf{p} \\
\mathbf{t}
\end{array}\right) R_{\mathbf{t q}}\right) \mid r \geq 0, R \in \mathscr{R}_{\mathbf{r}}, \mathbf{p}, \mathbf{q} \in \mathscr{G}^{r}\right\rangle
$$

becomes a biideal of $\mathfrak{H}(\mathscr{G})$, the quotient $\mathfrak{H}=\mathfrak{H}(\mathscr{G} / \mathscr{R}):=\mathfrak{H}(\mathscr{G}) / \mathfrak{I}$ becomes a $\mathscr{V}$-face algebra. Let $g$ be a group-like element of $\mathfrak{S}(\mathscr{G} / \mathscr{R})$. Let $X_{\mathbf{p q}}^{(r)}\left(\mathbf{p}, \mathbf{q} \in \mathscr{G}^{r}\right.$, $r \geq 0$ ) be the image of $e\left(\begin{array}{l}\mathbf{p} \\ \mathbf{q}\end{array}\right) \in \mathfrak{H}(\mathscr{G})$ in $\mathfrak{H}$ and $Y=\left[Y_{\mathbf{p q}}\right]_{\mathbf{p}, \mathbf{q} \in \mathscr{G} 1}$ an element of Mat $\left(\mathscr{G}^{1}, \mathfrak{H}\right)$. We say that $Y$ is a cofactor matrix of $X=\left[X_{\mathbf{p q}}^{(1)}\right]$ with respect to $g$ if the following relations are satisfied:

$$
\begin{aligned}
& Y X=P g, X Y=Q g, \\
& P Y=Y=Y Q,
\end{aligned}
$$

where $P$ and $Q$ denote elements of Mat $\left(\mathscr{G}^{1}, \mathscr{G}\right)$ defined by

$$
\begin{aligned}
& P=P^{(1)}, Q=Q^{(1)} \\
& P_{\mathbf{p q}}^{(r)}:=\delta_{\mathbf{p q}} e_{\imath(\mathbf{p})}, Q_{\mathbf{p q}}^{(r)}:=\delta_{\mathbf{p q}} \stackrel{\circ}{\lrcorner}_{\lrcorner(\mathbf{p})} \quad\left(\mathbf{p}, \mathbf{q} \in \mathscr{G}^{r}, r \geq 0\right) .
\end{aligned}
$$

Theorem 7.3. $(c f .[\mathrm{T}])$ If there exists a cofactor matrix $\left[Y_{\mathbf{p q}}\right]$ with respect to $g$, then $g$ is central and the quotient $\overline{\mathfrak{H}}=\mathfrak{H}(\mathscr{G} / \mathscr{R}) /(g-1)$ is a Hopf face algebra with antipode

$$
S\left(\bar{X}_{\mathbf{p q}}\right)=\bar{Y}_{\mathbf{p q}},
$$

where $^{-}: \mathfrak{H}(\mathscr{G} / \mathscr{R}) \rightarrow \overline{\mathfrak{H}}$ denotes the natural projection.

Proof. Using $X P=X=Q X$ and $g Q=Q g$, we compute

$$
\begin{aligned}
X g & =X P g=X Y X \\
& =g Q X=g X .
\end{aligned}
$$

Since $\mathfrak{H}$ is generated by $\left\{e\left(\begin{array}{l}i \\ j\end{array}\right), X_{\mathbf{p q}}\right\}$, this implies that $g$ belongs to the center of $\mathfrak{S}$. For $r \geq 0$, we define a matrix $Y^{(r)} \in \operatorname{Mat}\left(\mathscr{G}^{r}, \mathfrak{H}\right)$ by

$$
Y_{i j}^{(0)}=e\left(\begin{array}{l}
j \\
i
\end{array}\right) \quad\left(i, j \in \mathscr{G}^{0}=\mathscr{V}\right)
$$




$$
\begin{aligned}
Y_{\mathbf{p q}}^{(r)}=Y_{\mathbf{p}_{r} \mathbf{q}_{r}} \cdots & Y_{\mathbf{p}_{2} \mathbf{q}_{2}} Y_{\mathbf{p}_{1} \mathbf{q}_{1}} \\
& \left(\mathbf{p}=\left(\mathbf{p}_{1}, \cdots, \mathbf{p}_{r}\right), \mathbf{q}=\left(\mathbf{q}_{1}, \cdots, \mathbf{q}_{r}\right) \in \mathscr{G}^{r}, r \geq 1\right) .
\end{aligned}
$$

Then, we obtain the following relations:

$$
\begin{aligned}
& Y^{(r)} X^{(r)}=P^{(r)} g^{r}, X^{(r)} Y^{(r)}=Q^{(r)} g^{r}, \\
& X^{(r)} P^{(r)}=X^{(r)}=Q^{(r)} X^{(r)}, \\
& P^{(r)} Y^{(r)}=Y^{(r)}=Y^{(r)} Q^{(r)} .
\end{aligned}
$$

Let $R$ be an element of $\mathscr{R}_{r}$. Using these formulas, we compute

$$
\begin{aligned}
\overline{Y^{(r)} R} & =\overline{Y^{(r)} Q^{(r)} R}=\overline{Y^{(r)} R Q^{(r)}}=\overline{Y^{(r)} R X^{(r)} Y^{(r)}} \\
& =\overline{Y^{(r)} X^{(r)} R Y^{(r)}}=\overline{P^{(r)} R Y^{(r)}}=\overline{R Y^{(r)}}
\end{aligned}
$$

Hence there exists a linear map $\tilde{S}: \mathfrak{H} \rightarrow \overline{\mathfrak{H}}$ such that $\tilde{S}\left(X_{\mathbf{p q}}^{(r)}\right)=\overline{Y_{\mathbf{p q}}^{(r)}}$. Using $\widetilde{S}\left(a \stackrel{\circ}{i}_{i}\right)=e_{i} \tilde{S}(a)(a \in \mathfrak{H}, i \in \mathscr{V})$ and

$$
\sum_{(a)} \overline{a_{(1)}} \tilde{S}\left(a_{(2)}\right)=\sum_{i \in \mathscr{V}} \varepsilon\left(e_{i} \bar{a}\right) \stackrel{\circ}{e}_{i} \quad(a \in \mathfrak{H})
$$

we obtain

$$
1=\sum_{i \in \mathcal{V}} \varepsilon\left(e_{i} \bar{g}\right) \stackrel{\circ}{e}_{i}=\sum_{i \in \mathcal{V}} \overline{g e}_{i} \tilde{S}\left(g \stackrel{\circ}{e}_{i}\right)=\tilde{S}(g)
$$

Therefore (7.5) gives a well-defined map which is an antipode of $\overline{\mathfrak{S}}$.

Now we return to the situation of $\S 6$. We define a matrix $b_{J} \in \operatorname{Mat}\left(\mathscr{G}^{2}, \mathbb{C}\right)$ by

$$
\left(b_{J}\right)_{\mathbf{p} \cdot \mathbf{q}, \mathbf{r} \cdot \mathbf{s}}=W\left(\mathbb{p}_{\mathbf{q}}^{\mathbb{P}} \mathbf{s}\right) \quad\left(\mathbf{p} \in \mathscr{G}_{i m}^{1}, \mathbf{q} \in \mathscr{G}_{m n}^{1}, \mathbb{r} \in \mathscr{G}_{i j}^{1}, \mathbf{s} \in \mathscr{G}_{j n}^{1}\right) .
$$

Then, $\mathfrak{H}=\mathfrak{H}\left(\mathscr{G} /\left\{b_{J}\right\}\right)$ has a group-like element det, and $\mathfrak{F}(\mathscr{G})$ can be identified with the quotient $\mathfrak{H} /(\mathrm{det}-1)$ (see the proof of Proposition 4.2 of [H2]). Explicitly, det is given by

$$
\begin{aligned}
\operatorname{det} & =\sum_{i \in \mathcal{V}} \sum_{\mathbf{s} \in \mathscr{G}^{1}}\left(\frac{\mu(i) \mu(\iota(\mathbf{s}))}{\mu(\jmath(\mathbf{s})) \mu\left(\iota\left(\mathbf{p}_{i}\right)\right)}\right)^{1 / 2} e\left(\begin{array}{c}
\mathbf{p}_{i} \cdot \mathbf{p}_{i}^{\sim} \\
\mathbf{s} \cdot \mathbf{s}^{\sim}
\end{array}\right) \\
& =\sum_{j \in \mathcal{V}} \sum_{\mathbf{s} \in \mathscr{G}^{1}}\left(\frac{\mu(j) \mu(\iota(\mathbf{s}))}{\mu(\sigma(\mathbf{s})) \mu\left(\iota\left(\mathbf{p}_{j}\right)\right)}\right)^{1 / 2} e\left(\begin{array}{c}
\mathbf{s} \cdot \mathbf{s}^{\sim} \\
\mathbf{p}_{j} \cdot \mathbf{p}_{j}^{\sim}
\end{array}\right),
\end{aligned}
$$

where $\mathbf{p}_{i}$ denotes an arbitrary element of $\amalg_{k} \mathscr{G}_{i, k}^{1}$. Since 


$$
Y=\left[\left(\frac{\mu(\Im(\mathbf{q})) \mu(\iota(\mathbf{p}))}{\mu(J(\mathbf{p})) \mu(\iota(\mathbf{q}))}\right)^{1 / 2} e\left(\begin{array}{c}
\mathbf{q}^{\sim} \\
\mathbf{p}^{\sim}
\end{array}\right)\right]_{\mathbf{p}, \mathbf{q}}
$$

is a cofactor matrix with respect to det, $\mathfrak{F}(\mathscr{G})$ has an antipode $S$ given by (6.1). In fact, we have obtained the following result.

Lemma 7.4. Let $\mathscr{G}$ be a connected non-oriented graph which has at least one adge. Let $\mathfrak{H}(\mathscr{G} / \mathscr{R})$ and $b_{J}$ be as above. If $b_{J} \in \mathscr{R}$, then $\mathfrak{H}(\mathscr{G} / \mathscr{R})$ has a central group-like element det given by (7.6) and the quotient $\mathfrak{H}(\mathscr{G} / \mathscr{R}) /(\operatorname{det}-1)$ has an antipode given by (6.1).

\section{References}

[A] Abe, E., Hopf algebras, Cambridge tracts in mathematics 74, Cambridge Univ. Press., Cambridge, 1980.

[D] Drinfeld, V., Quantum groups, Proc. ICM, Berkeley, (1986), 798-820.

[ER] Effros, E. G. and Ruan, Z. J., Discrete quantum groups, I. The Haar measure, preprint.

[H1] Hayashi, T., An algebra related to the fusion rules of Wess-Zumino-Witten models, Lett. Math. Phys., 22 (1991), 291-296.

[H2] - Quantum group symmetry of partition functions of IRF models and its application to Jones' index theory, Commun. Math. Phys., 157 (1993), 331-345.

[H3] - Face algebras and their Drinfeld doubles, Proceedings of Symposia in Pure Mathematics., 56 (1994) Part 2, 49-61.

[H4] - Face algebras I-A generalization of quantum group theory, preprint.

[H5] - Galois quantum groups of $\mathrm{II}_{1}$-subfactors, preprint.

[H6] - in preparation.

[K] Koornwinder, T., Compact quantum groups and $q$-special functions, preprint.

[KP] Kac, G. I. and Paljutkin, V. G., Finite ring groups, Trans. Moscow Math. Soc., (1966), 251-294.

[L] Larson, R., Characters of Hopf algebras, J. Algebra, 17 (1971), 352-368.

[LR] Larson, R. and Radford, D., Semisimple cosemisimple Hopf algebras, Amer. J. Math., 109 (1987), 187-195.

[RTF] Reshetikhin, N., Takhtadzhyan, L. and Faddeev, L., Quantization of Lie groups and Lie algebras, Algebra and Analysis, 1 (1989), 178-206, English translation: Leningrad Math. J., 1 (1990), 193-225.

[S] Sweedler, M., Hopf algebras, W. A. Benjamin Inc., New York, 1969.

[T] Takeuchi, M., Matric bialgebras and quantum groups, Israel J. Math., 72 (1990), 232-251.

[W] Woronowicz, S., Compact matrix pseudogroups, Comm. Math. Phys., 111 (1987), 613-665. 
